\title{
PLANTAS MEDICINAIS: FATORES DE INFLUÊNCIA NO CONTEÚDO DE METABÓLITOS SECUNDÁRIOS
}

\author{
Leonardo Gobbo-Neto e Norberto P. Lopes*
}

Departamento de Física e Química, Faculdade de Ciências Farmacêuticas de Ribeirão Preto, Universidade de São Paulo, Av. do Café, s/n, 14040-903 Ribeirão Preto - SP, Brasil

Recebido em 7/7/05; aceito em 11/4/06; publicado na web em 31/10/06

\begin{abstract}
MEDICINAL PLANTS: FACTORS OF INFLUENCE ON THE CONTENT OF SECONDARY METABOLITES. Since secondary metabolites represent a chemical interface between plants and surrounding environment, their syntheses are frequently affected by environmental conditions. Thus, variations in the total content and/or of the relative proportions of secondary metabolites in plants can take place. We review the main environmental factors that can streamline or alter the production or concentration of secondary metabolites in plants. How seasonality, circadian rhythm, developmental stage and age, temperature, water availability, UV radiation, soil nutrients, altitude, atmospheric composition and tissue damage influence secondary metabolism are discussed.
\end{abstract}

Keywords: variations in secondary metabolites; medicinal plants; chemical ecology.

\section{INTRODUÇÃO}

Desde o quarto século a.C. existem relatos de normas para a coleta de plantas medicinais. Os carrascos gregos, por ex., coletavam suas amostras do veneno cicuta (Conium maculatum) pela manhã, quando os níveis de coniina são maiores ${ }^{1,2}$. Variações temporais e espaciais no conteúdo total, bem como as proporções relativas de metabólitos secundários em plantas ocorrem em diferentes níveis (sazonais e diárias; intraplanta, inter- e intraespecífica) e, apesar da existência de um controle genético, a expressão pode sofrer modificações resultantes da interação de processos bioquímicos, fisiológicos, ecológicos e evolutivos ${ }^{3-7}$. De fato, os metabólitos secundários representam uma interface química entre as plantas e o ambiente circundante, portanto, sua síntese é freqüentemente afetada por condições ambientais ${ }^{8}$.

Os principais fatores que podem coordenar ou alterar a taxa de produção de metabólitos secundários estão expostos a seguir. Deve ser enfatizado, porém, que os estudos sobre influência destes fatores na produção de metabólitos secundários geralmente têm se limitado a um grupo restrito de espécies, predominantemente ocorrentes em regiões temperadas, muitas das quais são comercialmente importantes e podem ter sofrido fortes pressões seletivas antrópicas visando certas características desejadas. Seu comportamento, portanto, nem sempre é representativo de plantas selvagens ou de outros tipos de habitat.

Também deve ser ressaltado que, muitas vezes, as variações podem ser decorrentes do desenvolvimento foliar e/ou surgimento de novos órgãos concomitante a uma constância no conteúdo total de metabólitos secundários. Isto pode levar à menor concentração destes metabólitos por diluição, podendo, no entanto, resultar em maior quantidade total, devido ao aumento de biomassa ${ }^{9,10}$. Além disso, alguns dos fatores discutidos apresentam correlações entre si e não atuam isoladamente, podendo influir em conjunto no metabolismo secundário, como por ex.: desenvolvimento e sazonalidade; índice pluviométrico e sazonalidade; temperatura e altitude, entre outros.

*e-mail: npelopes@fcfrp.usp.br

\section{FATORES QUE INFLUENCIAM O CONTEÚDO DE METABÓLITOS SECUNDÁRIOS}

\section{Sazonalidade, ritmo circadiano e desenvolvimento}

A época em que uma droga é coletada é um dos fatores de maior importância, visto que a quantidade e, às vezes, até mesmo a natureza dos constituintes ativos não é constante durante o ano. São relatadas, por ex., variações sazonais no conteúdo de praticamente todas as classes de metabólitos secundários, como óleos essenciais ${ }^{11-15}$, lactonas sesquiterpênicas ${ }^{16,17}$, ácidos fenólicos ${ }^{16,18}$, flavonóides ${ }^{19-25}$, cumarinas $^{21}$, saponinas ${ }^{26-27}$, alcalóides ${ }^{1,28-31}$, tani$\operatorname{nos}^{32-36}$, graxas epicuticulares ${ }^{37}$, iridóides ${ }^{22,38,39}$, glucosinolatos ${ }^{40,41}$ e glicosídeos cianogênicos ${ }^{42,43}$.

Os casos mais freqüentemente relatados envolvem plantas e/ ou metabólitos empregados na terapêutica, podendo ser citados os seguintes exemplos: as folhas de Digitalis obscura apresentam as menores concentrações de cardenolídeos, como o lanatosídeo A (Figura 1d), na primavera e uma fase de rápido acúmulo no verão, seguida por uma fase de decréscimo no outono ${ }^{31}$; as concentrações de hipericina (Figura 1c) e pseudo-hipericina na erva de São João (Hypericum perforatum, utilizada no tratamento de depressões leves a moderadas) aumentam de cerca de 100 ppm no inverno para mais de 3000 ppm no verão ${ }^{44}$; nas folhas de Ginkgo biloba as concentrações de biflavonóides, como a ginkgetina (Figura 1f), constituintes ativos dos extratos utilizados para tratamento de desordens vasculares periféricas e cerebrais, também apresentam marcantes variações sazonais ${ }^{23}$; por outro lado, não há consenso quanto às variações no conteúdo dos ginkgolidos, devido à existência de resultados contraditórios na literatura ${ }^{45}$; nas raízes de Panax ginseng foi detectado um grande aumento na concentração de damarano-saponinas bioativas, tais como o ginsenosídeo (Figura $1 \mathrm{~g}$ ) no verão ${ }^{27}$; o conteúdo de valepotriatos, como o valtrato (Figura 1a) e derivados do ácido valerênico (Figura 1b), nas raízes de Valeriana officinalis (utilizada como sedativo moderado) também apresenta variações marcantes durante o ano ${ }^{46}$; os conteúdos de $C$-glicosídeos, $O$-glicosídeos e antraquinonas livres (metabólitos responsáveis pela atividade laxante da planta) nos brotos e folhas da cáscara sagrada Rhamnus purshiana flutuam marcadamente durante o ano ${ }^{47}$; no inverno, o ruibarbo (Rhei rhizoma) não contém 
antraquinonas, as quais começam a se formar com a chegada da estação quente, a partir da oxidação de antranóis ${ }^{47}$; em estudos que visam determinar a sazonalidade de taxóides, tendo como alvo principal o paclitaxel (Figura 1e - utilizado na terapêutica de câncer de ovário, entre outros) notam-se alterações no decorrer do ano na concentração destes compostos em Taxus brevifolia e T. baccata; porém, não é possível determinar um padrão de variação, devido a resultados conflitantes na literatura ${ }^{48-53}$. Resultados contrastantes também foram encontrados nos estudos envolvendo as antraquinonas de Aloe arborescens ${ }^{54,55}$.
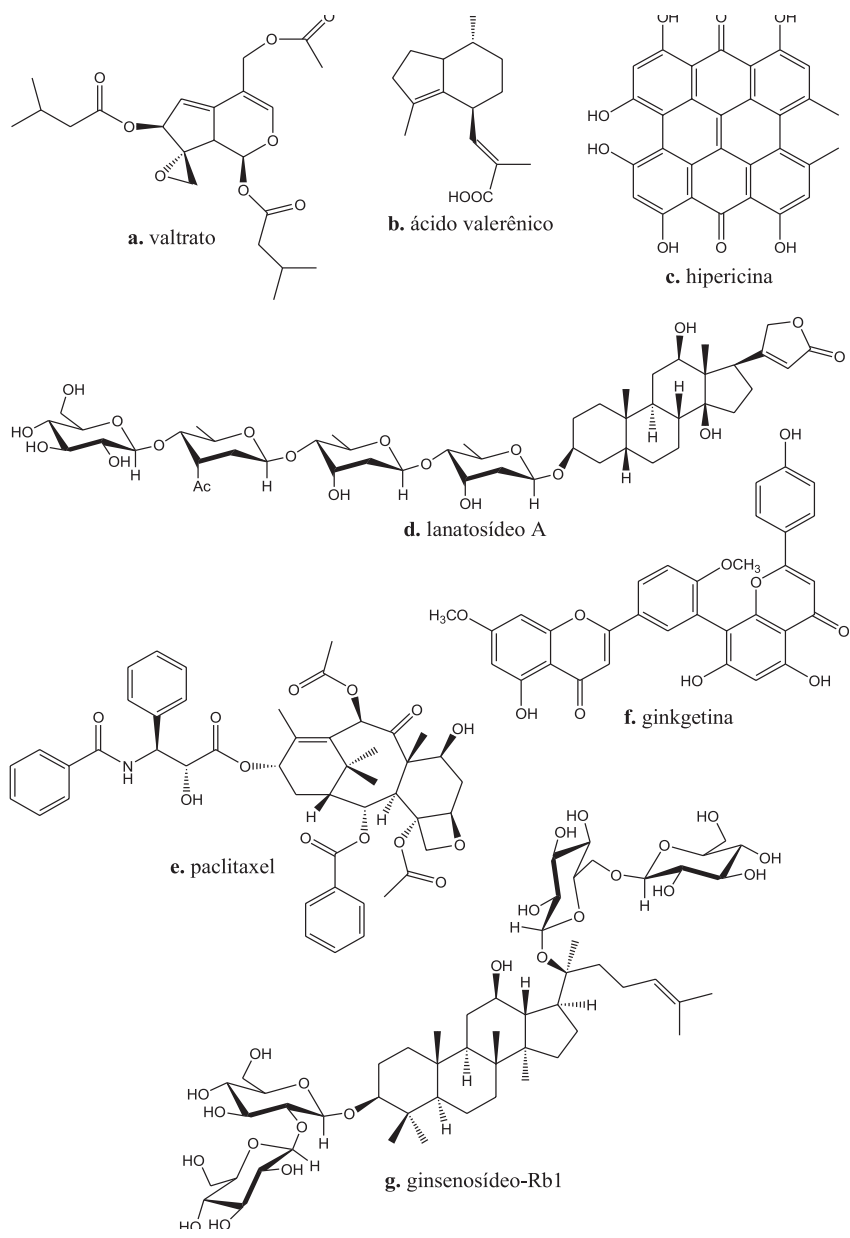

Figura 1. Exemplos de metabólitos secundários que podem ter seu acúmulo alterado por influência da sazonalidade

Existem, também, cada vez mais estudos mostrando que a composição de metabólitos secundários de uma planta pode variar apreciavelmente durante o ciclo dia/noite, tendo sido descritas, por ex., variações circadianas nas concentrações de óleos voláteis ${ }^{12,14,56-58}$, iridóides ${ }^{38}$, alcalóides ${ }^{1,2,59-61}$, glucosinolatos ${ }^{62}$, glicosídeos cianogênicos e tiocianatos ${ }^{63}$.

Foi notada, por ex., uma variação de mais de $80 \%$ na concentração de eugenol (Figura 2b) no óleo essencial da alfavaca (Ocimum gratissimum), o qual atinge um máximo em torno do meio-dia, horário em que é responsável por $98 \%$ do óleo essencial, em contraste com uma concentração de $11 \%$ em torno de $17 \mathrm{~h}^{56}$. Os níveis de coniina (Figura 2a) em Conium maculatum são maiores quando as coletas são efetuadas pela manhã que no entardecer $^{2}$. O conteúdo total de taxanos (por ex., Figura 1e) em Taxus media mostrou-se menor pela manhã, aumentando durante o dia e atingindo um máximo no final da noite ${ }^{51}$. Também é inte-

ressante notar que a variação circadiana dos alcalóides majoritários de Papaver somniferum, que era atribuída a um rápido "turnover" destes metabólitos, é na verdade causada por uma variação diária no conteúdo de água no látex da planta, o que leva a alterações na concentração, mas não no conteúdo total, de morfina (Figura 2c), codeína e noscapina ${ }^{59,60}$.
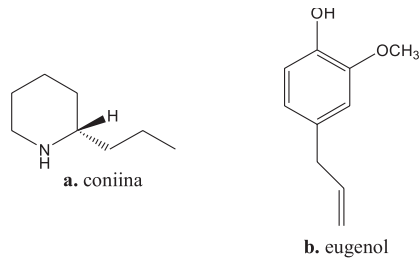

Figura 2. Exemplos de metabólitos secundários que podem ter seu acúmulo alterado por influência do ritmo circadiano

A idade e o desenvolvimento da planta, bem como dos diferentes órgãos vegetais, também são de considerável importância e podem influenciar não só a quantidade total de metabólitos produzidos, mas também as proporções relativas dos componentes da mistura ${ }^{5,10,47,64-74}$. É o caso, por ex., das lactonas sesquiterpênicas produzidas em Arnica montana, consideradas os principais princípios ativos desta planta utilizada como antiinflamatório; enquanto plantas jovens acumulam majoritariamente derivados da helenalina (Figura 3d), a concentração destes compostos é reduzida para praticamente zero após aproximadamente seis semanas contadas a partir da formação das folhas; por outro lado, os níveis de compostos do tipo diidrohelenalina aumentam muito e então se mantêm constantes por um longo período ${ }^{17}$. Situação semelhante ocorre com os metabólitos de Gentiana lutea, cujas folhas são ricas em $C$ glicosídeos, como a mangiferina (Figura 3b), na fase de floração, enquanto $O$-glicosídeos, como a isoorientina (Figura 3a), são acumulados principalmente antes do desenvolvimento das flores ${ }^{22}$. Em um estudo com Papaver somniferum (papoula), o conteúdo de morfina (Figura 2c) aumentou de menos de $20 \mu \mathrm{g} \mathrm{g}^{-1}$ no $50^{\circ}$ dia após a germinação para mais de $120 \mu \mathrm{g} \mathrm{g}^{-1}$ no $75^{\circ}$ dia; já o conteúdo de codeína se mostrou praticamente constante ${ }^{72}$. No caso do Tanacetum parthenium, a porcentagem de partenolídeo (Figura 3c - um dos principais constituintes bioativos desta planta utilizada principalmente na profilaxia da enxaqueca) é maior nos primeiros estágios de desenvolvimento da planta (antes do surgimento das hastes no ápice das quais surgirão as flores), porém a quantidade total obtida por planta aumenta de cerca de 10 para $20 \mathrm{mg}$ durante o crescimento, devido ao surgimento de flores (as quais, ao contrário das hastes, contêm mais de $1 \%$ de partenolídeo) e mais folhas ${ }^{10}$. Em Digitalis obscura micropropagada foi observado que os conteúdos de lanatosídeo A (Figura 1d - cardenolídeo predominante, responsável por cerca de $65 \%$ do total de cardenolídeos) e de digitoxina aumentam consideravelmente com o desenvolvimento da planta ${ }^{75}$.

Convém notar que, especialmente em estudos de campo e com plantas anuais, os efeitos da sazonalidade podem ser confundidos com alterações metabólicas sob controle do processo de desenvolvimento internamente (hormonalmente) controlado pela planta, devendo assim ser considerados em conjunto.

Sabe-se também que tecidos mais novos geralmente possuem maior taxa biossintética de metabólitos ${ }^{7}$, tais como óleos essenciais $^{76,77}$, lactonas sesquiterpênicas ${ }^{9}$, ácidos fenólicos ${ }^{78}$, alcalóides ${ }^{73}$, flavonóides e estilbenos ${ }^{71}$. De fato, nota-se freqüentemente uma correlação inversa entre alta atividade metabólica e produção de aleloquímicos, isto é, um decréscimo na produção de metabólitos secundários (notadamente derivados fenólicos) em períodos de crescimento tecidual rápido ${ }^{6,79-82}$. 
<smiles>O=c1cc(-c2ccc(O)c(O)c2)oc2cc(O)c(O[C@H]3O[C@H]4COC[C@H]([C@H](O)[C@H]4O)[C@H]3O)c(O)c12</smiles>

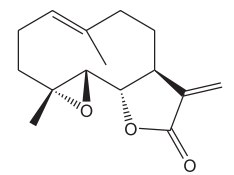

c. partenolídeo

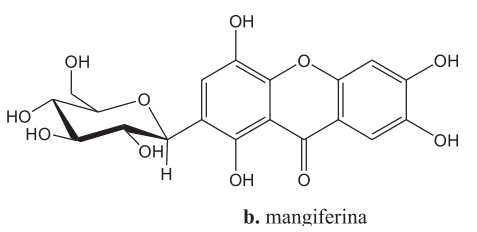

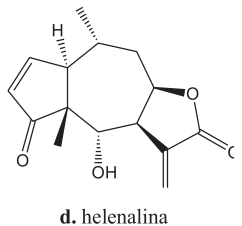

d. helenalina
Figura 3. Exemplos de metabólitos secundários cuja concentração na planta pode ser influenciada por sua idade ou estágio de desenvolvimento

\section{Temperatura}

Apesar de cada espécie ter se adaptado ao seu habitat, as plantas freqüentemente são capazes de existir em uma considerável faixa de temperatura. A faixa em que ocorrem as variações anuais, mensais e diárias na temperatura é um dos fatores que exerce maior influência em seu desenvolvimento, afetando, portanto, a produção de metabólitos secundários ${ }^{47}$. No entanto, talvez pelo fato da temperatura ser, de modo geral, uma consequiência de outros fatores, como altitude e sazonalidade, não existem muitos estudos sobre sua influência isoladamente na produção de metabólitos secundários.

As baixas temperaturas têm influências significantes nos níveis de metabólitos secundários. É relatada, por ex., uma correlação positiva entre a intensidade e a duração do frio imposto a mudas de milho (Zea mays) e a abundância de antocianinas e mRNA para as enzimas chaves da via fenilpropanoídica, tais como PAL (fenilalanina amônia-liase) e chalcona sintase ${ }^{83}$. Outro exemplo notório foi observado em Artemisia annua: após estresse metabólico causado por geada, verificou-se um aumento de cerca de $60 \%$ nos níveis de artemisinina (Figura 4b - substância com apreciável atividade contra cepas susceptíveis e resistentes de Plasmodium falciparum, agente causador da malária), concomitante a uma diminuição de seu precursor biossintético ácido diidroartemisínico, o que revelou uma rápida mobilização para a conversão deste em artemisinina ${ }^{84}$. Também foi demonstrado, em folhas de tabaco (Nicotiana tabacum), um aumento de quatro a cinco vezes no conteúdo de escopolina (Figura 4a), ácido clorogênico (Figura 4c) e seus isômeros (compostos antioxidantes) após submissão a baixas temperaturas ${ }^{78}$. $\mathrm{O}$ aumento nas concentrações de ácido clorogênico e antocianinas relacionados a baixas temperaturas também foram relatados em folhas de Mahonia repens, porém neste caso podem estar mais ligados à sazonalidade ${ }^{18}$.

A formação de óleos voláteis, em geral, parece aumentar em temperaturas mais elevadas, apesar de dias muito quentes levarem a uma perda excessiva destes metabólitos ${ }^{47}$. As concentrações de algumas substâncias componentes do óleo essencial de Santolina rosmarinifolia, entretanto, mostram uma correlação negativa com a temperatura ${ }^{13}$.

\section{Disponibilidade hídrica}

Fatores fisiológicos críticos, tais como fotossíntese, comportamento estomatal, mobilização de reservas, expansão foliar e crescimento, podem ser alterados por estresse hídrico e, conseqüentemente, levar a alterações no metabolismo secundário ${ }^{85-87}$.

Os efeitos da chuva na vegetação devem ser considerados em relação ao índice anual, sua distribuição pelo ano, seu efeito na umidade e seu efeito conjunto com a capacidade de absorção de
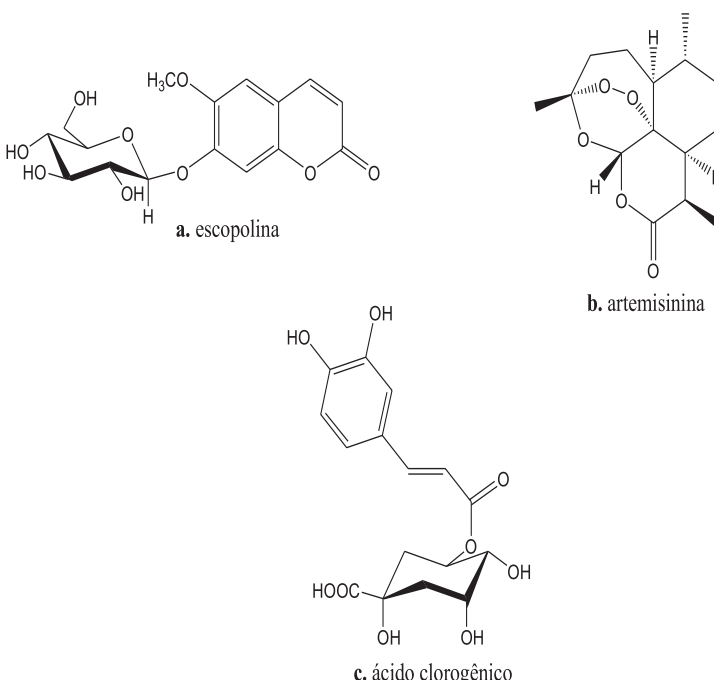

b. artemisinina

Figura 4. Exemplos de metabólitos secundários cuja concentração pode ser influenciada pela temperatura

água do solo $^{47}$. Exemplos da influência do índice pluviométrico na produção de metabólitos secundários são a correlação positiva de alguns dos componentes do óleo essencial de Santolina rosmarinifolia ${ }^{13}$ e a correlação negativa entre a produção de saponinas, como a lemmatoxina (Figura 5a) em Phytolacca dodecandra, com os níveis de precipitação ${ }^{26}$. Em Hypericum perforatum há um aumento significativo na concentração de flavonóides, hipericinas (Figura 1c) e ácido clorogênico (Figura 4c) nas flores sob condições de estresse hídrico; porém, há um decréscimo na concentração de hiperforinas (Figura $5 b)^{88}$.

Conforme mencionado, o estresse hídrico freqüentemente tem conseqüências significantes nas concentrações de metabólitos secundários em plantas, e há vários relatos de que estas condições geralmente levam a um aumento na produção de vários tipos de metabólitos secundários ${ }^{6,79}$, como glicosídeos cianogênicos, glucosinolatos ${ }^{89}$, alguns terpenóides ${ }^{90}$, antocianinas ${ }^{91}$ e alcalóides ${ }^{92,93}$. Com relação a metabólitos fenólicos, os estudos realizados apresentam resultados conflitantes e parece não ser possível estabelecer uma correlação clara entre sua concentração e estresse osmótico ${ }^{43,79,94-99}$, porém, nem sempre há alterações no acúmulo de metabólitos decorrentes de variações hídricas, como ocorre por ex. com os alcalóides de Catharanthus roseus ${ }^{100}$. O efeito da seca na concentração de metabólitos é, às vezes, dependente do grau de estresse e do período em que ocorre, sendo que efeitos
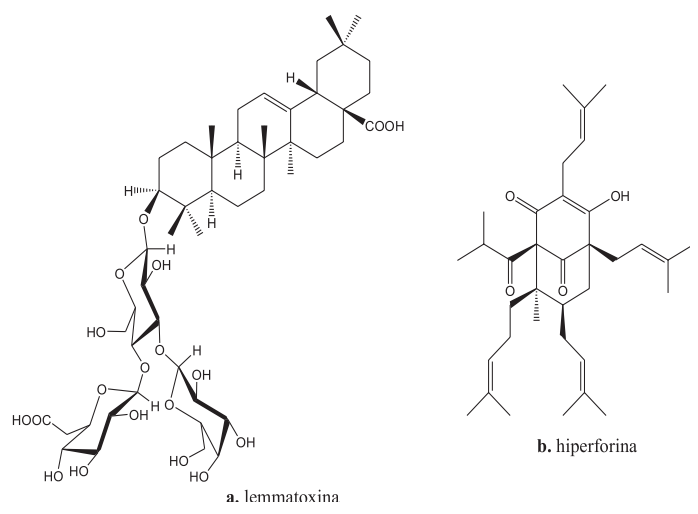

Figura 5. Exemplos de metabólitos secundários cujo acúmulo pode ser influenciado pela disponibilidade hídrica 
a curto prazo parecem levar a uma produção aumentada, enquanto a longo prazo é observado um efeito oposto $6,95,98,101,102$. Outro fator é que a chuva contínua pode resultar na perda de substâncias hidrossolúveis das folhas e raízes por lixiviação; sabe-se que isto se aplica a algumas plantas produtoras de alcalóides, glicosídeos e até mesmo óleos voláteis ${ }^{47,101}$.

\section{Radiação ultravioleta}

As diferentes espécies de plantas estão adaptadas a uma enorme variação na intensidade e quantidade de incidência luminosa ${ }^{6,47,85}$. Além disso, existe uma crescente preocupação com os efeitos do aumento de radiação ultravioleta (UV-B, 280-320 nm), decorrente da depleção da camada de ozônio.

Existe uma correlação positiva bem estabelecida entre intensidade de radiação solar e produção de compostos fenólicos ${ }^{101}$, tais como flavonóides $^{99,103,104}$, taninos ${ }^{94,105}$ e antocianinas ${ }^{18,106,107}$. Isso pode ser explicado, principalmente no caso de flavonóides e fenilpropanóides correlatos, pela proteção contra a foto-destruição proporcionada por estes metabólitos ao absorver e/ou dissipar a energia solar, dificultando assim a danificação dos tecidos mais internos pela radiação UV-B ${ }^{101,108-110}$.

No caso específico dos flavonóides, estes são acumulados principalmente em tecidos superficiais (tais como epiderme, subepiderme, pêlos, cutícula e material epicuticular) e utilizados pela planta como filtros UV, pois absorvem radiação UV-B sem alterar a radiação fotossinteticamente ativa ${ }^{101,111,112}$. Além disso, também podem atuar como "radical-scavengers" e antioxidantes ${ }^{108,113}$. O aumento na produção de tais metabólitos "protetores solares" é controlado por enzimas da rota biossíntética dos fenilpropanóides (fenilalanina amônia-liase e chalcona sintase, entre outras), as quais podem ter sua expressão gênica induzida pela luz ${ }^{114-119}$; no entanto, isto não se aplica a taninos e compostos fenólicos simples, os quais têm absorção máxima em comprimentos de onda consideravelmente mais curtos que os da UV-B ${ }^{101}$.

É interessante notar, também, o aumento na proporção de glicosídeos de luteolina (por ex., Figura 6b) / glicosídeos de apigenina (por ex., Figura 6c) quando do aumento de radiação UV$\mathrm{B}$ incidente em Marchantia polymorpha. Isto não aumenta a capacidade de absorção de UV-B mas, por outro lado, sabe-se que derivados da luteolina são mais efetivos que os da apigenina em dissipar a energia absorvida, aumentando deste modo os níveis de defe-
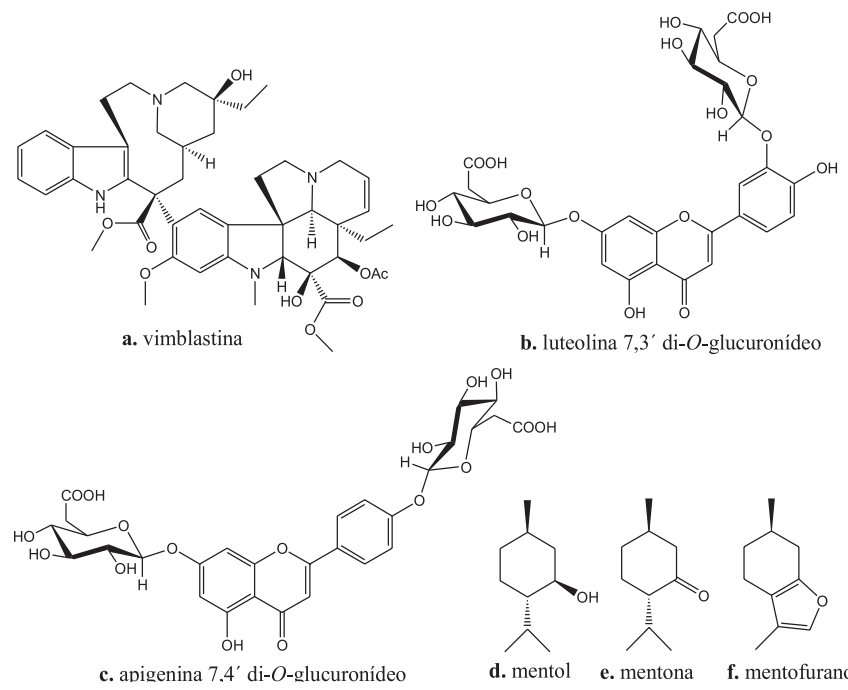

Figura 6. Exemplos de metabólitos secundários que podem ter seu acúmulo alterado por influência da intensidade elou quantidade de radiação ultravioleta sa antioxidante ${ }^{120}$. Situação semelhante foi recentemente descrita para a relação flavonóides/hidróxi-cinamatos, onde foi observado um aumento desta proporção por indução luminosa ${ }^{99}$. Isso demonstra que não somente a capacidade de absorver radiação UV-B, mas também a capacidade de atuar como antioxidantes, podem determinar a concentração e composição de metabólitos anti-UV-B ${ }^{99}$.

Estudos mostram que a intensidade de luz é um fator que também influencia a concentração e/ou composição de outras classes de metabólitos secundários ${ }^{6}$, como terpenóides ${ }^{9,121-123}$, glicosídeos cianogênicos $^{124}$ e alcalóides ${ }^{92-125}$. Foi demonstrado, por ex., que no manjericão (Ocimum basilicum) e no tomilho (Thymus vulgaris), duas plantas utilizadas na medicina popular e como condimentos, o completo desenvolvimento de tricomas glandulares, onde os óleos essenciais são armazenados, é luz-dependente ${ }^{123,126}$.

Em Atropa belladonna, Datura stramonium e Cinchona ledgeriana uma exposição total à luz solar leva a um maior conteúdo de alcalóides que a permanência na sombra ${ }^{47}$, comportamento oposto ao apresentado pelos alcalóides de Tabernaemontana pachysiphon $^{92}$. Hirata et al. ${ }^{125}$ demonstraram que a irradiação de Catharanthus roseus (vinca) com radiação UV na faixa entre 290$380 \mathrm{~nm}$ estimula a dimerização dos alcalóides catarantina e vindolina em vimblastina (Figura $6 \mathrm{a}$ - um antitumoral utilizado na terapêutica de vários tipos de cânceres) aumentando sua produção, bem como de outros alcalóides diméricos. A formação de lactonas sesquiterpênicas nos tricomas do girassol (Helianthus annuus) também é influenciada pela luz, sendo que uma exposição intensa leva a um maior nível biossintético ${ }^{9}$. As folhas de hortelã (Mentha piperita) mantidas sob condições de dia longo contêm mentol (Figura 6d), mentona (Figura 6e), e somente traços de mentofurano (Figura 6f), enquanto as plantas desenvolvidas sob condições de dia curto apresentam o mentofurano como constituinte majoritário do óleo essencial ${ }^{127}$.

\section{Nutrientes}

$\mathrm{Na}$ agricultura, a adição de nutrientes, particularmente nitrogênio, é geralmente empregada para aumentar a produção de biomassa. No entanto, os nutrientes afetam não somente o metabolismo primário, mas também influenciam a produção de diferentes metabólitos secundários, e o impacto de mudanças em sua disponibilidade na produção de metabólitos secundários foi revista por Gershenzon ${ }^{79}$. Estes efeitos, de certo modo, não são totalmente previsíveis; tendências podem ser reconhecidas, mas não é possível estabelecer regras sólidas e estáveis ${ }^{6}$. Por outro lado, apesar da reconhecida influência no desenvolvimento vegetal, poucos estudos mostram relações entre $\mathrm{pH}$ ou microorganismos do solo e metabolismo secundário ${ }^{47}$.

\section{Macronutrientes}

A grande maioria dos estudos sobre influência de nutrientes no solo está correlacionada com intensidade de luz incidente e voltada para discussões sobre alocação de recursos (hipóteses de balanço carbono/nutrientes), as quais visam estabelecer uma relação entre as disponibilidades de nitrogênio, carbono e luz ${ }^{128,131}$. Nestes estudos, de modo geral, a produção de metabólitos secundários (exceto os nitrogenados) mostra uma correlação positiva com a proporção carbono/nutrientes $(\mathrm{C} / \mathrm{N})$, isto é, em solos pobres em nutrientes, paralelamente à menor taxa de crescimento, geralmente se verifica maior produção de metabólitos secundários, particularmente derivados fenólicos ${ }^{79,94,129,130}$. Contudo, estas hipóteses não foram totalmente comprovadas, existindo resultados controversos e tentativas de refinamento das hipóteses de balanço $\mathrm{C} / \mathrm{N}^{34,128,130-134}$.

Observa-se que estresse nutricional usualmente resulta em aumento nas concentrações de metabólitos secundários, exceto no 
caso da deficiência de nitrogênio e enxofre, em que a produção de metabólitos secundários contendo estes elementos é diminuída ${ }^{79}$.

A produção global de metabólitos nitrogenados por uma planta (alcalóides, glicosídeos cianogênicos e glucosinolatos) geralmente é aumentada com a maior disponibilidade de nitrogênio no solo ${ }^{6,79,92}$. Entretanto, como consequiência do aumento da biomassa da planta, a concentração destes nos tecidos pode diminuir ${ }^{6,79}$. Além disso, existem evidências de que não é somente a disponibilidade ambiental de nitrogênio em si que influencia o metabolismo secundário, mas sim a quantidade deste que é incorporada aos tecidos da planta ${ }^{94}$. Em solos ácidos, devido a uma redução na taxa de conversão de amônio a nitrato, a incorporação de nitrogênio pode ser inibida, o que tem sido utilizado para explicar estudos que constataram altos níveis de produção de metabólitos secundários (especialmente compostos fenólicos) associados a plantas crescendo nesse tipo de solo $^{6}$. Os níveis de fósforo e potássio, apesar de relativamente pouco estudados, também podem ter efeitos na produção de metabólitos nitrogenados $6,47,79$.

Uma correlação bem estabelecida é que menores quantidades de metabólitos fenólicos são produzidas em condições de fornecimento abundante de nitrogênio ${ }^{135,136}$. Os efeitos de nutrientes nos níveis de derivados do ácido chiquímico (especialmente ácidos cinâmicos simples e taninos hidrolisáveis e condensados) são bem documentados e deficiências em nitrogênio, fósforo, enxofre e potássio geralmente resultam em maiores concentrações destes metabólitos ${ }^{6,79,94}$.

Por outro lado, os metabólitos derivados do mevalonato parecem não mostrar correlações consistentes com mudanças na disponibilidade de nitrogênio, fósforo ou potássio ${ }^{79}$.

\section{Micronutrientes}

Há pouca informação disponível sobre o impacto de micronutrientes na produção de metabólitos secundários em plantas. Foi mostrado, por ex., que o fornecimento de manganês e molibdênio, através do borrifamento das folhas com soluções de sais destes elementos, durante o desenvolvimento de Digitalis grandiflora (dedaleira) leva a um aumento de mais de duas vezes no conteúdo de heterosídeos cardioativos ${ }^{137}$. Outros estudos relacionando manganês ${ }^{75,138,139}$ e conteúdo de micronutrientes nas folhas ${ }^{140}$ à produção de cardioativos foram realizados, porém os resultados são contrastantes.

$\mathrm{O}$ tratamento de suspensões celulares de Catharanthus roseus com cádmio pode aumentar em mais de três vezes a produção de ajmalicina (Figura 9b), um dos alcalóides de interesse comercial da planta, e sua excreção para o meio de cultura ${ }^{141}$. Também foi relatado que o tratamento com cádmio ou cobre altera a excreção de alcalóides para o meio em cultura celular de raízes transformadas de Atropa belladonna $^{142}$. A falta de boro reduz a produção de compostos fenólicos em palmeiras ${ }^{143}$ e o fornecimento de estanho e bismuto aumenta a quantidade de taninos produzidos por Acacia catuch $u^{6}$.

\section{Altitude}

A altitude também exerce efeitos sobre o desenvolvimento e a produção de metabólitos secundários em plantas, apesar de existirem relativamente poucos estudos neste sentido. A correlação positiva geralmente existente entre o conteúdo total de flavonóides e a altitude, por ex., é de particular interesse farmacêutico, uma vez que estes são constituintes ativos de um grande número de plantas medicinais ${ }^{16,144-146}$. Esta correlação pode ser explicada pela maior susceptibilidade à radiação UV em altitudes maiores, uma vez que, conforme comentado anteriormente, os flavonóides são reconhecidos por propiciarem proteção à radiação e seus efeitos.

Foi descrita, por ex., uma significante correlação positiva entre a altitude do local de coleta (entre 30 e 2950 m) de espécies do gênero Leontodon e o conteúdo total de flavonóides; em contraste, o conteúdo de ácidos fenólicos não mostrou variação altitudinal significante ${ }^{16}$. Um maior conteúdo de compostos fenólicos em Sedum album coletada em altitudes maiores $(2100 \times 240 \mathrm{~m})$ também foi relatado $^{145}$. Por outro lado, estudo recente com Swertia franchetiana (matéria-prima para elaboração de um importante medicamento popular conhecido como DiDa no Tibet, utilizado no tratamento de uma série de doenças, como desordens do fígado) mostrou que, entre as altitudes de 2200 e 3960 m, dentre os metabólitos secundários bioativos analisados, somente as concentrações das xantonas mangiferina (Figura 3b) e 1,8-diidróxi-3,7-dimetóxi-xantona correlacionaram-se com a latitude, e apenas a última mostrou correlação (negativa, no caso) com a altitude ${ }^{147}$.

Metabólitos não fenólicos também podem ser influenciados pela altitude. Uma comparação entre coletas de Plantago lanceolata efetuadas em altas altitudes $x$ baixas altitudes revelou maiores concentrações do iridóide catalpol (Figura 7a) nas primeiras ${ }^{4}$. O aumento de altitude leva a um decréscimo no conteúdo dos alcalóides diterpênicos de Aconitum napellus e piperidínicos de Lobelia inflata e no de óleos voláteis de tomilho e hortelã pimenta ${ }^{47}$. Já o conteúdo de piretrinas (por ex. Figura $7 \mathrm{~b}$ - substâncias utilizadas comercialmente como inseticidas) em Chrysanthemum é maior e, altas altitudes e baixas latitudes ${ }^{47}$.
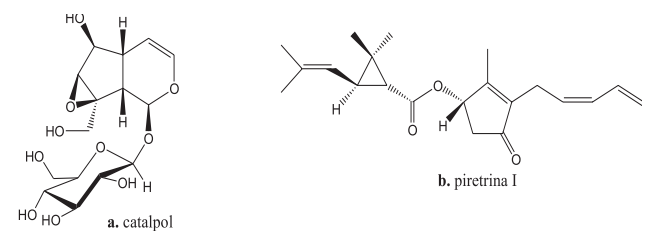

Figura 7. Exemplos de metabólitos secundários que podem ter seu acúmulo alterado por influência da altitude

\section{Poluição atmosférica}

Os poucos trabalhos sobre alterações no metabolismo secundário decorrentes de poluição atmosférica são, de modo geral, bastante limitados e voltados principalmente para as consequiências de níveis elevados de $\mathrm{O}_{3}$ ou de $\mathrm{CO}_{2}$ no metabolismo de derivados fenólicos.

A exposição de plantas de 6 semanas de idade de Pinus sylvestris a $\mathrm{O}_{3}$, na concentração de pelo menos $0,15 \mu \mathrm{L} \mathrm{L}^{-1}$ por $8 \mathrm{~h}$, resultou em forte indução (aumento de centenas de vezes, dose- e tempo-dependente) na formação dos estilbenos pinosilvina (Figura 8d) e pinosilvina-3-metiléter, assim como da atividade da enzima estilbenosintase. Por outro lado, os níveis de pinocembrina e catequina não se alteraram significativamente com este tratamento ${ }^{148}$. Alterações no conteúdo de alguns polifenóis, como $p$-hidróxi-acetofenona, piceína (Figura 8c) e 3-O-glicosil-kaempferol foram observados nas folhas de abeto (Picea abies), porém resultados conflitantes foram obti$\operatorname{dos}^{148,149}$. A fumigação das folhas de soja (Glycine max) com $\mathrm{O}_{3}$ (de 0,25 a $1 \mu \mathrm{L} \mathrm{L}^{-1}$ por $3 \mathrm{~h}$ ), paralelamente aos danos foliares, levou a um maior acúmulo dos isoflavonóides cumestrol (Figura 8a), daidzeína (Figura 8b) e sojagol; $\mathrm{NO}_{2}$ e $\mathrm{SO}_{2}$ também causaram um acúmulo, porém bem menor, destes isoflavonóides ${ }^{150}$.

Foi relatado que Digitalis lanata cultivada em casas de vegetação com atmosfera enriquecida com $\mathrm{CO}_{2}(1000 \mathrm{ppm})$ produziu 3,5 vezes mais digoxina (Figura 8e - um heterosídeo cardioativo utilizado na terapêutica da insuficiência cardíaca congestiva) por hectare que plantas cultivadas no campo ${ }^{47}$. Por outro lado, Plantago lanceolata quando cultivada em atmosfera com elevada concentração de $\mathrm{CO}_{2}\left(700 \mu \mathrm{L} \mathrm{L}^{-1}\right)$ apresenta concentrações similares ou menores de iridóides que a cultivada em condição ambiente $\left(\mathrm{CO}_{2}=350 \mu \mathrm{L} \mathrm{L}^{-1}\right)^{131}$. 


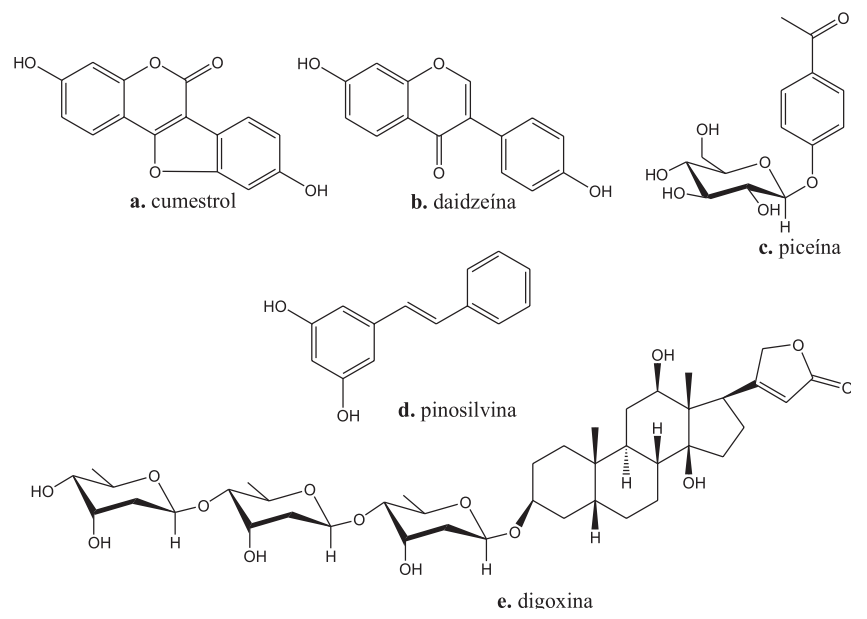

Figura 8. Exemplos de metabólitos secundários que podem ter sua concentração alterada por influência da composição atmosférica

Também há alguns relatos de mudanças quali- e quantitativas em resinas e aumento na emissão de terpenóides voláteis resultantes de tratamentos com $\mathrm{SO}_{2}$ ou $\mathrm{O}_{3}{ }^{151}$.

\section{Indução por estímulos mecânicos ou ataque de patógenos}

Fatores mecânicos aos quais as plantas estão susceptíveis, tais como ferimentos, ou mesmo meros estímulos, causados por chuva, granizo, vento, areia, invasão por patógenos e pastagem de herbívoros, também podem influenciar a expressão do metabolismo secundário ${ }^{5,141,153,154}$.

Danos causados a plantas por ferimentos ou ataque de herbívoros ou patógenos freqüentemente levam a uma resposta bioquímica, que reduz a aceitabilidade do órgão ou de todo o organismo a ataques futu$\operatorname{ros}^{6,155}$. O mais claro exemplo disto é a produção de novo de fitoalexinas (compostos geralmente derivados de fenilpropanóides, mas também de terpenóides ou poliacetilenos) em resposta à invasão de patógenos $^{6,85,155,156}$. Algumas plantas, como tomate e batata, em resposta à alimentação por insetos produzem rapidamente peptídeos inibidores de proteinase, o que pode diminuir significantemente a palatabilidade da planta ou parte dela ${ }^{6,157-159}$.

Uma outra forma de defesa induzida, aparentemente bem distinta da anterior, é a resposta a curto ou longo prazo à danificação de tecidos vegetais aumentando a produção e o acúmulo de metabólitos secundários já existentes na planta, levando à fuga dos animais $^{6,158}$. Este acréscimo é, às vezes, uma resposta restrita ao órgão danificado, e outras vezes uma resposta mais geral, podendo afetar a bioquímica vegetal como um todo ${ }^{6,160,161}$.

A danificação artificial em plântulas de Catharanthus roseus, por ex., resultou em um aumento de cerca de $100 \%$ no conteúdo dos alcalóides ajmalicina (Figura 9b) e vindolina 48 h após o tratamento; em contraste, o conteúdo de catarantina não sofreu alterações significativas ${ }^{153}$. O acúmulo de alcalóides indólicos monoterpênicos em vinca também se mostrou estimulado pela danificação em folhas jovens, enquanto não foram observadas alterações metabólicas em folhas maduras, o que sugere um maior comprometimento implícito da proteção química com os tecidos mais jovens em relação aos já desenvolvidos ${ }^{100}$.

Variando o número de ferimentos artificiais e quantificando o ácido jasmônico resultante após 90 min e a concentração de nicotina (Figura 9a) na planta após 5 dias, foi encontrada uma correlação positiva entre número de ferimentos nas folhas, ácido jasmônico endógeno e acúmulo de nicotina em toda a planta ${ }^{160}$. Sabe-se que o ácido jasmônico é um regulador de rotas biossintéticas e responsá- vel por parte da rota sinalizadora que aumenta notavelmente a síntese de novo de nicotina nas raízes de Nicotiana sylvestris, de onde ela é distribuída para toda a planta ${ }^{155,160}$. Portanto, um distúrbio físico em um local específico pode proporcionar um sinal suficiente para induzir respostas em locais distantes e não estimulados ${ }^{160}$.

$\mathrm{O}$ ferimento artificial das folhas do arbusto Eriodictyon californicum resultou em uma diminuição de até $63 \%$ no conteúdo de resinas fenólicas das folhas; porém, a composição da resina não mostrou alterações ${ }^{161}$. Em Asclepias syriaca (uma das plantas das quais a borboleta monarca seqüestra os cardenolídeos tóxicos que utiliza como defesa), o acúmulo destas substâncias é rapidamente induzido, atingindo um máximo 24 h após a danificação das folhas e diminuindo praticamente aos níveis constitutivos após $148 \mathrm{~h}^{162}$. Similarmente, McCloud et al. ${ }^{163}$ verificaram que as cucurbitacinas da aboboreira são rapidamente induzidas $1 \mathrm{~h}$ após a danificação e retornam aos níveis constitutivos menos de $10 \mathrm{~h}$ após. Outros relatos interessantes são os aumentos no conteúdo de taninos ${ }^{164} \mathrm{e}$ iridóides ${ }^{5}$ em resposta à danificação de folhas por lagartas.

Sabe-se também que o nível de indução pode variar de acordo com o agente causador. De fato, algumas observações têm revelado que injúria causada por alimentação de insetos pode resultar em uma resposta fisiológica diferenciada, na qual a expressão gênica e/ou formação de metabólitos secundários é induzida especificamente ou mais rapidamente, devido a eliciadores presentes na saliva do inseto ${ }^{152,165,166}$.

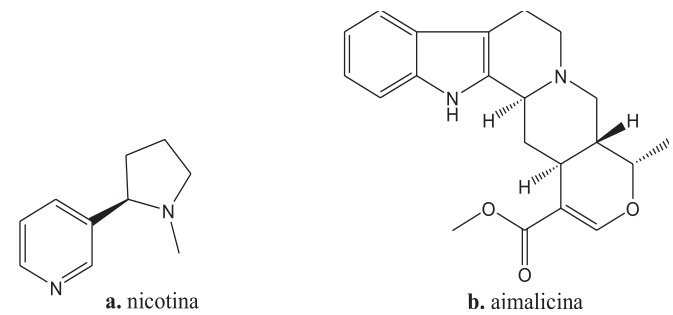

Figura 9. Exemplos de metabólitos secundários que podem ter seu acúmulo alterado por influência de estímulos mecânicos

\section{CONCLUSÕES}

Conforme visto, o metabolismo secundário de plantas pode variar consideravelmente dependendo de vários fatores (Figura 10), sendo que a constância de concentrações de metabólitos secundários é praticamente uma exceção. Por outro lado, estudo recente mostrou que os metabólitos secundários de uma espécie vegetal selvagem, amostrada diretamente em seu habitat em três diferentes populações, se mantiveram em concentrações constantes durante os dois anos do estudo, demonstrando, portanto, que em alguns casos o metabolismo secundário pode não se alterar em função de fatores climáticos, temporais ou ambientais ${ }^{167}$.

Os fatores expostos aqui (Figura 10), bem como outros que podem afetar o conteúdo final de metabólitos secundários em plantas medicinais, tais como condições de coleta, estabilização e estocagem, podem ter grande influência na qualidade e, conseqüentemente no valor terapêutico de preparados fitoterápicos ${ }^{47,168}$. O controle de qualidade e a padronização de fitoterápicos envolvem várias etapas, entretanto, a fonte e a qualidade das matérias-primas têm um papel central na obtenção de produtos com constância de composição e propriedades terapêuticas reprodutíveis ${ }^{168}$. O aprimoramento e o investimento em estudos de domesticação, produção biotecnológica e melhoramentos genéticos de plantas medicinais, ao invés do uso de plantas selvagens coletadas diretamente no campo, deve levar à obtenção de matérias-primas uniformes e de alta qualidade ${ }^{47,168}$.

O processo legal de regulamentação e legislação de preparados fitoterápicos, devido principalmente a aspectos culturais, é dife- 


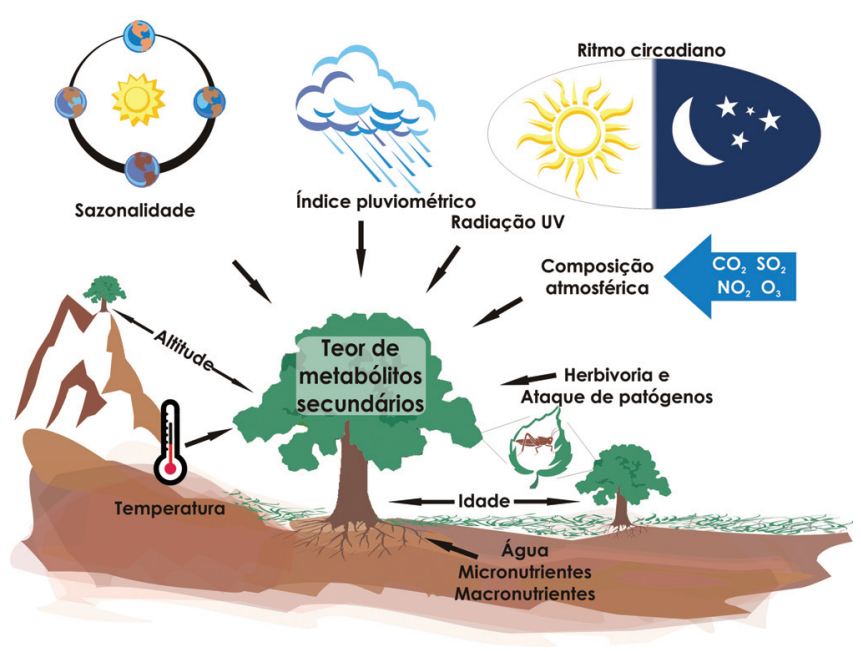

Figura 10. Principais fatores que podem influenciar o acúmulo de metabólitos secundários em planta

rente em cada país. Muitos países da Europa utilizam plantas medicinais de forma ampla, como a Alemanha e a França que detêm 39 e $29 \%$ do total de vendas da União Européia, respectivamente ${ }^{169,170}$. As legislações européias têm sido ampliadas por normas cada vez mais restritivas, exigindo testes que comprovem a eficácia, qualidade e segurança dos fitoterápicos ${ }^{169,170}$.

Neste mesmo sentido, no Brasil, resoluções recentes da Agência Nacional de Vigilância Sanitária (ANVISA) dispõem sobre o registro de medicamentos fitoterápicos ${ }^{171}$. A Resolução-RDC n ${ }^{\circ} 48$, de 16/3/ 2004 exige, com exceção para aqueles fitoterápicos enquadrados como “fitoterápicos tradicionais", a apresentação de uma série de relatórios que atestem, para o preparado fitoterápico a ser registrado, a segurança e a eficácia, bem como normas de produção e controle de qualidade visando reprodutibilidade e constância de princípios ativos e/ ou marcadores característicos da espécie vegetal ${ }^{169,171}$.

Baseando-se nos relatos aqui apresentados, é evidente que além da importância dos estudos de adulterações e contaminações ${ }^{169}$ existe a necessidade de uma análise química detalhada de plantas destinadas ao uso terapêutico. Sabendo-se dos inúmeros fatores que podem levar a variações no conteúdo de metabólitos secundários, fica clara a necessidade de estudos visando detectar as condições e épocas para cultivo e/ou coleta que conduzam a uma matéria-prima vegetal com concentrações desejáveis de princípios ativos. Também se faz necessário um rigoroso controle de qualidade realizado por meio de técnicas analíticas modernas, para garantir constância na composição de metabólitos secundários no preparado fitoterápico em escala industrial. Além disso, o reconhecimento e a compreensão dessas variações poderão, no futuro, auxiliar na ampliação dos conhecimentos sobre interações ecológicas do vegetal com seu ambiente.

\section{AGRADECIMENTOS}

Ao pós-graduando C. A. Carollo, DFQ-FCFRP, pela colaboração na elaboração das figuras, e ao $\mathrm{CNPq}$ pelas bolsas concedidas aos autores.

\section{REFERÊNCIAS}

1. Robinson, T.; Science 1974, 184, 430.

2. Fairbairn, J. W.; Suwal, P. N.; Phytochemistry 1961, 1, 38.

3. Lindroth, R. L.; Hsia, M. T. S.; Scriber, J. M.; Biochem. Syst. Ecol. 1987, 15, 681.

4. Darrow, K.; Bowers, M. D.; Biochem. Syst. Ecol. 1997, 25, 1.
5. Bowers, M. D.; Stamp, N. E.; Ecology 1993, 74, 1778.

6. Waterman, P. G.; Mole, S. Em Insect-plant interactions; Bernays, E. A., ed.; $1^{\text {st }}$ ed., CRS Press: Boca Raton, 1989, vol. 1, cap. 4.

7. Hartmann, T.; Ent. Exp. Appl. 1996, 80, 177.

8. Kutchan, T. M.; Plant Physiol. 2001, 125, 58.

9. Spring, O.; Bienert, U.; J. Plant Physiol. 1987, 130, 441.

10. Hendriks, H.; Anderson-Wildeboer, Y.; Engels, G.; Bos, R.; Woerdenbag, H. J.; Planta Med. 1997, 63, 356.

11. Schwob, I.; Bessiere, J. M.; Masotti, V.; Viano, J.; Biochem. Syst. Ecol. 2004, 32,735 .

12. Angelopoulou, D.; Demetzos, C.; Perdetzoglou, D.; Biochem. Syst. Ecol. 2002, 30, 189.

13. Palá-Paúl, J.; Pérez-Alonso, M. J.; Velasco-Negueruela, A.; Palá-Paúl, R.; Sanz, J.; Conejero, F.; Biochem. Syst. Ecol. 2001, 29, 663.

14. Lopes, N. P.; Kato, M. J.; Andrade, E. H. A.; Maia, J. G. S.; Yoshida, M.; Phytochemistry 1997, 46, 689.

15. Pitarevic., I.; Kuftinec, J.; Bla•evic. ， N.; Kuštrak, D.; J. Nat. Prod. 1984, 47, 409.

16. Zidorn, C.; Stuppner, H.; Taxon 2001, 50, 115.

17. Schmidt, T. J.; Bomme, U.; Alfermann, A. W.; Planta Med. 1998, 64, 268.

18. Grace, S. C.; Logan, B. A.; Adams III, W. W.; Plant Cell Environ. 1998, 21, 513.

19. Brooks, J. S.; Feeny, P.; Biochem. Syst. Ecol. 2004, 32, 769.

20. Atkinson, P.; Blakeman, J. P.; New Phytol. 1982, 92, 63.

21. Wilt, F. M.; Miller, G. C.; Biochem. Syst. Ecol. 1992, 20, 53.

22. Menkovic., N.; Savikin-Fodulovic., K.; Savin, K.; Planta Med. 2000, 66, 178.

23. Lobstein, A.; Jako, L. R.; Berrurier, M. H.; Anton, R.; Planta Med. 1991, 57, 430.

24. Clark, L. E.; Clark, W. D.; Biochem. Syst. Ecol. 1990, 18, 145.

25. Jalal, M. A. F.; Read, D. J.; Haslam, E.; Phytochemistry 1982, 21, 1397.

26. Ndamba, J.; Lemmich, E.; Mølgaard, P.; Phytochemistry 1994, 35, 95.

27. Kim, S. K.; Sakamoto, I.; Morimoto, K.; Sakata, M.; Yamasaki, K.; Tanaka, O.; Planta Med. 1981, 42, 181.

28. Elgorashi, E. E.; Drewes, S. E.; Staden, J. V.; Fitoterapia 2002, 73, 490.

29. Wu, T. S.; Wang, M. L.; Wu, P. L.; Phytochemistry 1996, 43, 785.

30. Bandopadhyay, J.; De, B.; Int. J. Pharmacog. 1997, 35, 349.

31. Roca-Pérez, L.; Boluda, R.; Gavidia, I.; Pérez-Bermúdez, P.; Phytochemistry 2004, 65, 1869.

32. Salminen, J. P.; Ossipov, V.; Haukioja, E.; Pihlaja, K.; Phytochemistry 2001, $57,15$.

33. Osier, T. L.; Hwang, S. Y.; Lindroth, R. L.; Biochem. Syst. Ecol. 2000, 28, 197.

34. Iason, G. R.; Hartley, S. E.; Duncan, A. J.; Biochem. Syst. Ecol. 1993, 21, 315.

35. Jonasson, S.; Bryant, J. P.; Chapin III, F. S.; Anderson, M.; Am. Nat. 1986, $128,394$.

36. Feeny, P. P.; Bostock, H.; Phytochemistry 1968, 7, 87.

37. Faini, F.; Labbé, C.; Coll, J.; Biochem. Syst. Ecol. 1999, 27, 673.

38. Høgedal, B. D.; Mølgaard, P.; Biochem. Syst. Ecol. 2000, 28, 949.

39. Bowers, M. D.; Collinge, S. K.; Gamble, S. E.; Schmitt, J.; Oecologia 1992, 91, 201.

40. Agerbirk, N.; Olsen, C. E.; Nielsen, J. K.; Phytochemistry 2001, 58, 91.

41. Rodman, J. E.; Louda, S. M.; Biochem. Syst. Ecol. 1985, 13, 405.

42. Kaplan, M. A. C.; Figueiredo, M. R.; Gottlieb, O. R.; Biochem. Syst. Ecol. 1983, $11,367$.

43. Cooper-Driver, G. A.; Finch, S.; Swain, T.; Bernays, E.; Biochem. Syst. Ecol. 1977, 5, 211.

44. Southwell, I. A.; Bourke, C. A.; Phytochemistry 2001, 56, 437.

45. Beek, T. A.; Bombardelli, E.; Morazzoni, P.; Peterlongo, F.; Fitoterapia 1998, $59,195$.

46. Bos, R.; Woerdenbag, H. J.; Putten, F. M. S.; Hendriks, H.; Scheffer, J. J. C.; Planta Med. 1998, 64, 143.

47. Evans, W. C.; Trease and Evans' Pharmacognosy, $14^{\text {th }}$ ed., WB Saunders Company: London, 1996, cap. 7.

48. Hook, I.; Poupat, C.; Ahond, A.; Guénard, D.; Guéritte, F.; Adeline, M. T.; Wang, X. P.; Dempsey, D.; Breuillet, S.; Potier, P.; Phytochemistry 1999, 52, 1041.

49. Veselá, D.; 'aman, D.; Valterová, I.; Vank, T.; Phytochem. Anal. 1999, 10, 319.

50. Vance, N. C.; Kelsey, R. G.; Sabin, T. E.; Phytochemistry 1994, 36, 1241.

51. ElSohly, H. N.; Croom, E. M.; Kopycki, W. J.; Joshi, A. S.; McChesney, J. D.; Phytochem. Anal. 1997, 8, 124.

52. Glowniak, K.; Mroczek, T.; Zobel, A. M.; Phytomedicine 1999, 6, 135.

53. Wheeler, N. C.; Jech, K.; Masters, S.; Brobst, S. W.; Alvarado, A. B.; Hoover, A. J.; Snader, K. M.; J. Nat. Prod. 1992, 55, 432.

54. Beppu, H.; Kawai, K.; Shimpo, K.; Chihara, T.; Tamai, I.;Ida, C.; Ueda, M.; Kuzuya, H.; Biochem. Syst. Ecol. 2004, 32, 783.

55. Park, M. K.; Park, J. H.; Kim, N. Y.; Shin, Y. G.; Choj, Y. S.; Lee, J. G.; Kim, K. H.; Lee, S. K.; Phytochem. Anal. 1998, 9, 186. 
56. Silva, M. G. V.; Craveiro, A. A.; Matos, F. J. A.; Machado, M. I. L.; Alencar, J. W.; Fitoterapia 1999, 70, 32.

57. Loughrin, J. H.; Kemp, T. R. H.; Burton, H. R.; Andersen, R. A.; Phytochemistry 1993, 32, 1417

58. Loughrin, J. H.; Hamilton-Kemp, T. R.; Andersen, R. A.; Hildebrand, D. F; Phytochemistry 1990, 29, 2473.

59. Itenov, K.; Mølgaard, P.; Nyman, U.; Phytochemistry 1999, 52, 1229.

60. Fairbairn, J. W.; Wassel, G.; Phytochemistry 1964, 3, 253.

61. Sporer, F.; Sauerwein, M.; Wink, M.; Acta Hortic. 1993, 331, 379

62. Rosa, E. A. S.; Heaney, R. K.; Rego, F. C.; Fenwick, G. R.; J. Sci. Food Agric. 1994, 66, 457.

63. Okolie, P. N.; Obasi, B. N.; Phytochemistry 1993, 33, 775.

64. Jenks, M. A.; Tuttle, H. A.; Feldmann, K. A.; Phytochemistry 1996, 42, 29.

65. Buta, J. G.; Spaulding, D. W.; J. Plant Growth Regul. 1997, 16, 43.

66. Doan, A. T.; Ervin, G.; Felton, G.; Biochem. Syst. Ecol. 2004, 32, 117.

67. Vogt, T.; Gülz, G.; Phytochemistry 1994, 36, 591.

68. Scora, R. W., Kumamoto, J.; Horner, P. F.; Hollenberg, J. L.; J. Nat. Prod. 1984, 47, 279.

69. Wisdom, C. S.; Rodriguez, E.; Biochem. Syst. Ecol. 1983, 11, 345.

70. Goralka, R. J. L.; Schumaker, M. A.; Langenheim, J. H.; Biochem. Syst Ecol. 1996, 24, 93.

71. Slimestad, R.; Biochem. Syst. Ecol. 1998, 26, 225.

72. Williams, R. D.; Ellis, B. E.; Phytochemistry 1989, 28, 2085.

73. Höft, M.; Verpoorte, R.; Beck, E.; Planta Med. 1998, 64, 148

74. Wilkinson, R. E.; Kasperbauer, M. J.; Phytochemistry 1972, 11, 2439

75. Gavidia, I; Pérez-Bermúdez, P.; Phytochemistry 1997, 45, 81.

76. Hall, G. D.; Langenheim, J. H.; Biochem. Syst. Ecol. 1986, 14, 61.

77. Gershenzon, J.; Maffei, M.; Croteau, R.; Plant Physiol. 1989, 89, 1351.

78. Koeppe, D. E.; Rohrbaugh, L. M.; Rice, E. L.; Wender, S. H.; Physiol. Plant. 1970, 23, 258.

79. Gershenzon, J.; Rec. Adv. Phytochem. 1984, 18, 273.

80. Crankshaw, D. R.; Langenheim, J. H.; Biochem. Syst. Ecol. 1981, 9, 115.

81. Palo, R. T.; Sunnerheim, K.; Theander, O.; Oecologia 1985, 65, 314.

82. Lilov, D.; Angelova, Y.; Biol. Plant. 1987, 29, 34

83. Christie, P. J.; Alfenito, M. R.; Walbot, V.; Planta 1994, 194, 541.

84. Wallaart, T. E.; Pras, N.; Beekman, A. C.; Quax, W. J.; Planta Med. 2000, 66, 57.

85. Salisbury, F. B.; Ross, C. W.; Plant Physiology, $4^{\text {th }}$ ed., Wadsworth Publishing Co.: Belmont, 1991.

86. Hsiao, T. C.; Annu. Rev. Plant Phys. 1973, 24, 519.

87. Bazaaz, F.; Chiariello, N.; Coley, P.; Pitelka, L.; Bioscience 1987, 37, 58.

88. Gray, D. E.; Pallardy, S. G.; Garret, H. E.; Rottinghaus, G. E.; Planta Med. 2003, 69, 1024

89. Blua, M. J.; Hanscom, Z.; Collier, B. D.; J. Chem. Ecol. 1998, 14, 623.

90. Lokar, L. C.; Maurich, V.; Mellerio, G.; Moneghini, M.; Poldini, L.; Biochem. Syst. Ecol. 1987, 15, 327.

91. Jung, S.; Plant Sci. 2004, 166, 459.

92. Höft, M.; Verpoorte, R.; Beck, E.; Oecologia 1996, 107, 160.

93. Briske, D. D.; Camp, B. J.; Weed Sci. 1982, 30, 106.

94. Dustin, C. D.; Cooper-Driver, G. A.; Biochem. Syst. Ecol. 1992, 20, 99

95. Horner, J. D.; Biochem. Syst. Ecol. 1990, 18, 211.

96. Dement, W. A.; Mooney, H. A.; Oecologia 1974, 15, 65

97. Guinn, G.; Eidenbock, M. P.; Crop Sci. 1982, 22, 614.

98. Mattson, W. J.; Haack, R. A.; Bioscience 1987, 37, 110.

99. Tattini, M.; Galardi, C.; Pinelli, P.; Massai, R.; Remorini, D.; Agati, G.; New Phytol. 2004, 163, 547.

100. Frischknecht, P. M.; Battig, M.; Baumann, T. W.; Phytochemistry 1987, 26, 707.

101. Waterman, P. G.; Mole, S.; Analysis of phenolic plant metabolites, $1^{\text {st }}$ ed., Blackwell Scientific Publications: Oxford, 1994, cap. 3

102. Medina, E.; Olivares, E.; Diaz, M.; Oecologia 1986, 70, 441.

103. Cuadra, P.; Harborne, J. B.; Waterman, P. G.; Phytochemistry 1997, 45, 1377.

104. Markham, K. R.; Tanner, G. J.; Caasi-Lit, M.; Whitecross, M. I.; Nayudu, M.; Mitchell, K. A.; Phytochemistry 1998, 49, 1913.

105. Dudt, J. F.; Shure, D. J.; Ecology 1994, 75, 86.

106. Jeong, S. T.; Goto-Yamamoto, N.; Kobayashi, S.; Esaka, M.; Plant Sci. 2004, 167, 247 .

107. Paré, P. W.; Tumlinson, J. H.; Plant Physiol. 1997, 114, 1161.

108. Grace, S. C.; Logan, B. A.; Phil. Trans. R. Soc. Lond. B 2000, 355, 1499.

109. Delucia, E. H.; Day, T. A.; Vogelmann, T. C.; Plant Cell Environ. 1992 15,921 .

110. Ålenius, C. M.; Vogelmann, T. C.; Bornman, J. F.; New Phytol. 1995, 131, 297.

111. Bieza, K.; Lois, R.; Plant Physiol. 2001, 126, 1105

112. Caldwell, M. M. R.; Robberecht, R.; Flint, S. D.; Physiol. Plant. 1983, $58,445$.

113. Larson, R. A.; Arch. Insect Biochem. Physiol. 1995, 29, 175

114. Chappell, J.; Hahlbrock, K.; Nature 1984, 311, 76.

115. Logemann, E.; Tavernara, A.; Schulz, W.; Somssich, I. E.; Hahlbrock, K.; Proc. Natl. Acad. Sci. U.S.A 2000, 97, 1903.
116. Jones, D. H.; Phytochemistry 1984, 23, 1349.

117. Zucker, M.; Annu. Rev. Plant Physiol. 1972, 23, 133.

118. Schmelzer, E.; Jahnen, W.; Hahlbrock, K.; Proc. Natl. Acad. Sci. U.S.A 1988, 85, 2989.

119. Bharti, A. K.; Khurana, J. P.; Photochem. Photobiol. 1997, 65, 765.

120. Markham, K. R.; Ryan, K. G.; Bloor, S. J.; Mitchell, K. A.; Phytochemistry 1998, 48, 791.

121. Karousou, R.; Grammatikopoulos, G.; Lanaras, T.; Manetas, Y.; Kokkini, S.; Phytochemistry 1998, 49, 2273.

122. Johnson, C. B.; Kirby, J.; Naxakis, G.; Pearson, S.; Phytochemistry 1999, $51,507$.

123. Yamaura, T.; Tanaka, S.; Tabata, M.; Phytochemistry 1989, 28, 741

124. Burns, A. E.; Gleadow, R. M.; Woodrow, I. E.; Oecologia 2002, 133, 288

125. Hirata, K.; Asada, M.; Yatani, E.; Miyamoto, K.; Miura, Y.; Planta Med. 1993, 59,46

126. Ioannidis, D.; Bonner, L.; Johnson, C. B.; Ann. Bot. 2002, 90, 453.

127. Voirin, B.; Brun, N.; Bayet, C.; Phytochemistry 1990, 29, 749.

128. Koricheva, J.; Larsson, S.; Haukioja, E.; Keinänen, M.; Oikos 1998, 83, 212.

129. Coley, P. D.; Bryant, J. P.; Chapin III, F. S.; Science 1985, 230, 895.

130. Bryant, J. P.; Chapin III, F. S.; Klein, D. R.; Oikos 1983, 40, 357

131. Fajer, E. D.; Bowers, M. D.; Bazzaz, F. A.; Am. Nat. 1992, 140, 707.

132. Jones, C. G.; Hartley, S. E.; Oikos 1999, 86, 27.

133. Koricheva, J.; Oikos 2002, 98, 537

134. Herms, D. A.; Mattson, W. J.; Q. Rev. Biol. 1992, 67, 283.

135. Bryant, J. P.; Chapin III, F. S.; Reichardt, P. B.; Clausen, T. P.; Oecologia 1987, 72, 510

136. Price, P. W.; Waring, G. L.; Julkunen-Tiitto, R.; Tahvanainen, J.; Mooney, H. A.; Craig, T. P.; J. Chem. Ecol. 1989, 15, 1117.

137. Letchamo, W.; Planta Med. 1986, 5, 421.

138. Hagimori, M.; Matsumoto, T.; Obi, Y.; Agric. Biol. Chem. 1983, 47, 565.

139. Ohlsson, A.; Berglund, T. J.; J. Plant Physiol. 1989, 135, 505.

140. Roca-Pérez, L.; Boluda, R.; Pérez-Bermúdez, P.; J. Plant. Nutr. Soil Sci. 2004, 167, 79

141. Zheng, Z.; Wu, M.; Plant Sci. 2004, 166, 507.

142. Lee, K. T.; Yamakawa, T.; Kodama, T.; Shimomura, K.; Phytochemistry 1998, 49, 2343.

143. Rajaratnam, J. A.; Hook, L. I.; Exp. Agric. 1975, 11, 59.

144. Polle, A.; Mössnang, M.; Schönborn, A.; Sladkovic, R.; Rennenberg, H.; New Phytol. 1992, 121, 89.

145. Bachereau, F.; Marigo, G.; Asta, J.; Physiol. Plant. 1998, 104, 203.

146. Veit, M.; Bilger, W.; Mühlbauer, T.; Brummet, W.; Winter, K.; J. Plant Physiol. 1996, 148, 478.

147. Yang, H.; Duan, Y.; Hu, F.; Liu, J.; Biochem. Syst. Ecol. 2004, 32, 861.

148. Rosemann, D.; Heller, W.; Sandermann, H.; Plant Physiol. 1991, 97, 1280.

149. Heller, W.; Rosemann, D.; Osswald, W. F.; Benz, B.; Schönwitz, R.; Lohwasser, K.; Kloos, M.; Sandermann, H.; Environ. Pollut. 1990, 64, 353.

150. Keen, N. T.; Taylor, O. C.; Plant Physiol. 1975, 55, 731.

151. Riemer, J.; Whittaker, J. B. Em ref. 6, cap. 3.

152. Reymond, P.; Weber, H.; Damond, M.; Farmer, E. E.; The Plant Cell 2000, 12,707 .

153. Vázquez-Flota, F.; Carrilo-Pech, M.; Minero-Garcia, Y.; Miranda-Ham, M. L.; Plant Physiol. Biochem. 2004, 42, 623.

154. Cipollini Jr., D. F.; Oecologia 1997, 111, 84

155. Heil, M.; Bostock, R. M.; Annals of Botany 2002, 89, 503.

156. Harborne, J. B.; Introduction to ecological biochemistry, $3^{\text {rd }}$ ed., Academic Press Limited: London, 1989, cap. 10.

157. Graham, J. S.; Hall, G.; Pearce, G.; Ryan, C. A.; Planta 1986, 169, 399.

158. Harborne, J. B. Em ref. 156, cap. 7.

159. Hilder, V. A.; Gatehouse, A. M. R.; Sheerman, S. E.; Barker, R. F.; Boulter, D.; Nature 1987, 330, 160

160. Baldwin, I. T.; Zhang, Z. P.; Diab, N.; Ohnmeiss, T. E.; McCloud, E. S.; Lynds, G. Y.; Schmelz, E. A.; Planta 1997, 201, 397.

161. Johnson, N. D.; Brain, S. A.; Biochem. Syst. Ecol. 1985, 13, 5.

162. Malcolm, S. B.; Zalucki, M. P.; Ent. Exp. Appl. 1996, 80, 193.

163. McCloud, E. S; Tallamy, D. W.; Halaweish, F. T.; Ecol. Entomol. 1995, $20,51$.

164. Schultz, J. C.; Baldwin, I. T.; Science 1982, 217, 149.

165. Korth, K. L.; Dixon, R. A.; Plant Physiol. 1997, 115, 1299.

166. Neuvonen, S.; Haukioja, E.; Molarius, A.; Oecologia 1987, 74, 363.

167. Sakamoto, H. T.; Gobbo-Neto, L.; Cavalheiro, A. J.; Lopes. N. P.; Lopes, J. L. C.; J. Braz. Chem. Soc. 2005, 16, 1396.

168. Calixto, J. B.; Braz. J. Med. Biol. Res. 2000, 33, 179

169. Veiga Jr., V. F.; Pinto, A. C.; Maciel, M. A. M.; Quim. Nova 2005, 28, 519.

170. Silano, M.; De Vincenzi, M.; De Vincenzi, A.; Silano, V.; Fitoterapia 2004, $75,107$.

171. http://e-legis.bvs.br/leisref/public/showAct.php?id=10230, acessada em Novembro 2005 